\title{
Characterization of porous, dexamethasone-releasing polyurethane coatings for glucose sensors
}

\author{
Suzana G. Vallejo-Heligon ${ }^{\mathrm{a}}$, Bruce Klitzman ${ }^{\mathrm{a}, \mathrm{b}}$, William M. Reichert ${ }^{\mathrm{a}, *}$ \\ a Department of Biomedical Engineering, Duke University, Durham, NC 27708-0281, USA \\ ${ }^{\mathrm{b}}$ Kenan Plastic Surgery Research Labs, Duke University Medical Center, Durham, NC 27710, USA
}

\section{A R T I C L E I N F O}

\section{Article history:}

Received 29 March 2014

Received in revised form 15 July 2014

Accepted 18 July 2014

Available online 25 July 2014

\section{Keywords:}

Biosensor

Controlled drug release

Porosity

Foreign body response

Inflammation

\begin{abstract}
A B S T R A C T
Commercially available implantable needle-type glucose sensors for diabetes management are robust analytically but can be unreliable clinically primarily due to tissue-sensor interactions. Here, we present the physical, drug release and bioactivity characterization of tubular, porous dexamethasone (Dex)releasing polyurethane coatings designed to attenuate local inflammation at the tissue-sensor interface. Porous polyurethane coatings were produced by the salt-leaching/gas-foaming method. Scanning electron microscopy and micro-computed tomography (micro-CT) showed controlled porosity and coating thickness. In vitro drug release from coatings monitored over 2 weeks presented an initial fast release followed by a slower release. Total release from coatings was highly dependent on initial drug loading amount. Functional in vitro testing of glucose sensors deployed with porous coatings against glucose standards demonstrated that highly porous coatings minimally affected signal strength and response rate. Bioactivity of the released drug was determined by monitoring Dex-mediated, dose-dependent apoptosis of human peripheral blood derived monocytes in culture. Acute animal studies were used to determine the appropriate Dex payload for the implanted porous coatings. Pilot short-term animal studies showed that Dex released from porous coatings implanted in rat subcutis attenuated the initial inflammatory response to sensor implantation. These results suggest that deploying sensors with the porous, Dex-releasing coatings is a promising strategy to improve glucose sensor performance.
\end{abstract}

(c) 2014 Acta Materialia Inc. Published by Elsevier Ltd. All rights reserved.

\section{Introduction}

The dominant management strategy for blood sugar control in type I diabetes mellitus involve a combination of blood glucose monitoring by finger pricking and manual insulin delivery. These strategies are often inadequate in cases where tight glycemic control is prescribed [1-4]. Consequently, there is a pressing need for a closed-loop system in which real-time changes in glucose levels monitored by a sensor are used to regulate automated insulin delivery $[1,2,4,5]$. Unfortunately, contemporary implantable needle-type glucose sensors that could be used to manage insulin supply can behave unpredictably in vivo.

Often, a sensor that performs robustly and accurately in vitro may upon implantation continue to work adequately, fail acutely, show a steady drift, or exhibit a combination thereof. Interestingly, upon post-removal testing, the sensor will regain proper functionality [6-8]. This observation suggests that the unpredictable

\footnotetext{
* Corresponding author.

E-mail address: reichert@duke.edu (W.M. Reichert).
}

behavior of implanted sensors may be driven by the tissue-sensor interaction and not by failures in the sensor itself.

Implanted glucose sensors are subject to a dramatically varying tissue microenvironment over the 5-7 day period for which they are approved for patient use. Upon implantation, the sensor is presented with hemostasis followed by immune cell recruitment and inflammation, and finally the tissue gives way to a repair/remodeling stage comprised of provisional matrix formation, fibrosis and loss of vasculature. Several excellent reviews are available on this topic $[7,9]$. Adequately surviving this sequela of events, often referred to as the break-in period, has become an important design criterion in the development of implantable glucose sensors.

Initial strategies to extend sensor functionality have focused on preventing protein adsorption and cell attachment through the incorporation of hydrogel coatings $[10,11]$. The emerging sentiment is that resistance to biofouling is necessary but not sufficient to ensure proper sensor function. Numerical modeling and in vitro studies have recently shown that increased glucose consumption and enzymatic attack by immune cells during inflammation may be one of the dominant factors negatively affecting glucose sensor function [12-14]. 
Currently there exist essentially two schools of thought towards addressing acute inflammation: management and attenuation. Proponents of inflammation management view inflammation as a necessary step to achieve a stable and acceptable tissue bed for an implanted glucose sensor. Approaches that manage acute inflammation are inherently more complex and employ strategies to guide immune cell phenotype and cytokine production $[15,16]$. Attenuation of inflammation contends that the benefits of minimizing the deleterious effects of acute inflammation on sensor function outweigh the potential advantages of engineering the tissue response. One strategy for the attenuation of acute inflammation involves the local release of anti-inflammatory mediators such as nitric oxide, non-steroidal anti-inflammatory drugs and glucocorticoids [17-19].

Recent reports have shown that localized delivery of dexamethasone (Dex) reduces of anomalous sensor effects that arise from inflammatory cell invasion to the surface of an indwelling sensor [20]. Dex is a potent glucocorticoid associated with diminished activation of immune cells and up-regulation of anti-inflammatory cytokines [11,21-24]. However, localized delivery of Dex is often accompanied by decreased vascularity at the sensor-tissue interface [25]. Previous studies have also demonstrated that highly porous coatings of controlled structure ( $50-75 \mu \mathrm{m}$ pore size) could be used to increase vascular perfusion of the tissue bed [26,27]; to our knowledge researchers have yet to explore the combination of these two proven effects as a strategy to improve indwelling glucose sensor function.

Our goal is to incorporate Dex release as an inflammation attenuation component into a textured coating designed to increase long-term vascular density around implanted sensor leads. Therefore, the current study looked at the possibility of combining proangiogenic texturing with anti-inflammatory Dex release.

Here, we present the fabrication and characterization of Dexreleasing porous polyurethane coatings for needle-type glucose sensors. Pore size, porosity and coating thickness of the porous polyurethane coatings were evaluated by scanning electron microscopy (SEM) and micro-computed tomography (micro-CT). Signal strength and response time of sensors deployed with porous coatings was demonstrated using glucose standards. Drug release from porous coatings was monitored over 2 weeks as a function of initial loading and coating porosity. Bioactivity of the released drug was demonstrated by monitoring the Dex-mediated, dosedependent apoptosis of human peripheral blood derived monocytes in culture. Acute animal studies were used to determine the appropriate Dex payload for the implanted porous coatings. Pilot short-term animal studies showed that Dex released from porous coatings diminished the initial inflammatory response to glucose sensor implantation. These results suggest that deploying sensors with these porous, Dex-releasing coatings is a promising strategy to improve glucose sensor performance.

\section{Methods}

\subsection{Fabrication of Dex-releasing porous coatings}

Porous coatings were fabricated by the gas-foaming/salt-leaching technique described previously [27]. Briefly, a $6.5 \mathrm{wt} . \%$ solution of polyurethane was prepared by dissolving Tecoflex ${ }^{\circledR} 93 \mathrm{~A}$ pellets (Lubrizol Technologies) in a solution of 25:75 ethanol/chloroform. Dex (Sigma, D1756) was dissolved in the polymer solution and stirred until clear. Sieved $(50-75 \mu \mathrm{m})$ ammonium bicarbonate salt particles (MP Biomedicals, 150107) were added to the polymer solution and homogeneously mixed. Table 1 lists the amount of Dex and ammonium bicarbonate porogen added to polyurethane stock solutions to produce various specimen compositions.
Table 1

Porogen and Dex added to stock polymer solution to produce coatings with different porosities and Dex loadings.

\begin{tabular}{cccc}
\hline Intended \% porosity & Porogen $(\mathrm{g})$ & Intended wt.\% Dex & Dex $(\mathrm{mg})$ \\
\hline 90 & 1.5 & 2.9 & 180 \\
60 & 1.0 & 1.4 & 90 \\
30 & 0.5 & 0.7 & 45 \\
\hline
\end{tabular}

Dex-free porous and non-porous coatings were fabricated either by respectively not adding Dex or ammonium bicarbonate porogen to the polymer solution.

Polymer films were dip-coated onto copper wire mandrels (Belden, 20 AWG) and allowed to dry for $1 \mathrm{~h}$. Films were porated by placing the polymer-coated mandrels into deionized water for $5 \mathrm{~min}$ at $90^{\circ} \mathrm{C}$ to allow gas-foaming/salt-leaching to occur. Porated films were then quickly quenched in $4{ }^{\circ} \mathrm{C}$ deionized water for $20 \mathrm{~min}$. Films were allowed to dry in overnight in a desiccator, and cut to a length of $1.5 \mathrm{~cm}$ in order to fit over the sensing tips of Medtronic MiniMed SOF-SENSOR ${ }^{\mathrm{TM}}$ sensors (Fig. 1A).

\subsection{Scanning electron microscopy}

A vacuum sputter coater (Denton Desk IV) was used to deposit $10 \mathrm{~nm}$ gold layer on the surface of the porous coatings. SEM images of the porous coating surfaces and cross-sections were analyzed using ImageJ. A length measurement tool was generated based on the ratio of pixels per scale bar length of each image. Images were standardized to cover a $0.5 \mathrm{~mm}^{2}$ area of the coatings. Using the measurement tool, the major axis of each pore on the selected surface was measured (Fig. 1B). Cross-sectional images were used to measure the thickness of the coatings at the positions highlighted (Fig. 1C).

\subsection{Micro-computed tomography}

Porous coatings were soaked in Lugol's Iodine (EMS Cat. No. 26055 ) for 3 days and were vacuum dried overnight. Samples were evaluated using a Nikon XTH 225 ST micro-CT scanner. The X-ray source was set to $80 \mathrm{kV}$ and $120 \mu \mathrm{A}$, spot size $<3 \mu \mathrm{m}$, and rotation step $0.5^{\circ}$. An exposure time of $708 \mathrm{~ms}$ was set for each X-ray image. Four X-ray images were then averaged to obtain one 2-D projection. After acquisition, 2-D projections were reconstructed using CT Agent software to provide axial picture cross-sections. After reconstruction, the data was converted into 2000 16-bit picture files with a resolution of $<3 \mu \mathrm{m}$ per pixel. Complete volumes were rendered in Avizo Fire 8.0. Sub-volumes consisting of 500 z-stack images were selected for porosity evaluation. Sample porosity was calculated as the ratio of void to solid volume.



\subsection{Differential scanning calorimetry (DSC)}

Differential scanning calorimetry (DSC) (PerkinElmer, Diamond DSC N5360020) was employed to determine the physical state of Dex in the polyurethane coatings. Pre-weighed $10 \mathrm{mg}$ samples of Dex-loaded porous coatings were placed into aluminum pans and loaded into the sample chamber. Untreated Dex powder (Sigma, D1756) samples were used as controls. Control samples weighed $0.5 \mathrm{mg}$ to match the amount of Dex in contained in the $10 \mathrm{mg}$ Dex-loaded porous coating samples. The sample chamber was purged with nitrogen to prevent air oxidation of the samples at high temperatures. Samples were kept at $50{ }^{\circ} \mathrm{C}$ for $10 \mathrm{~min}$ during 


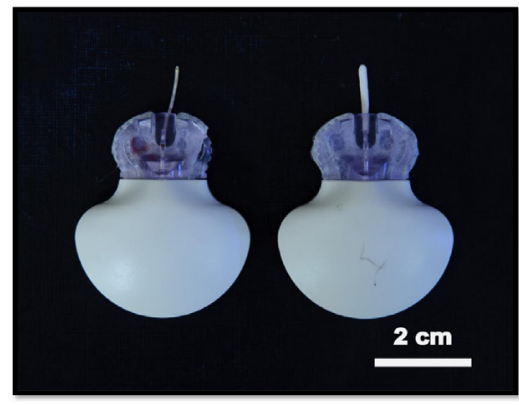

(A)

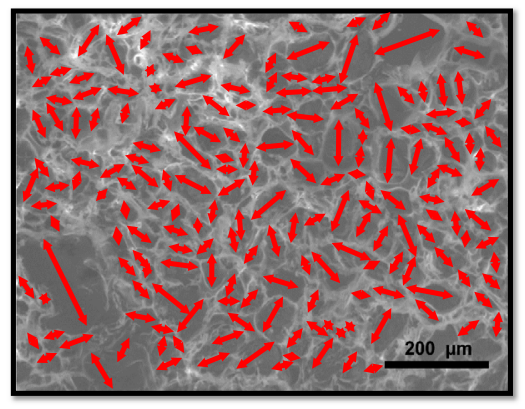

(B)

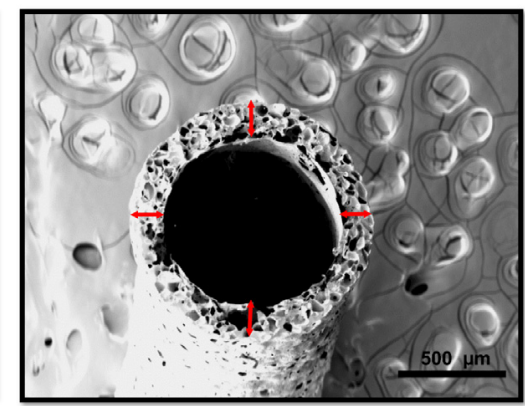

(C)

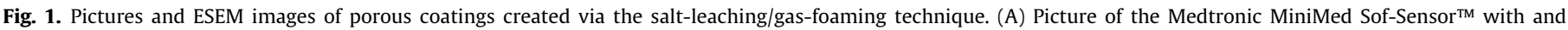

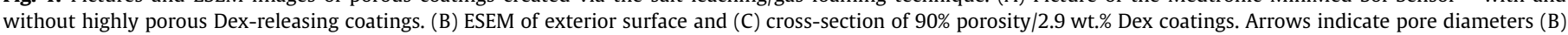
and coating thickness $(C)$. $(n=6$.).

preheating, and then samples were heated a constant rate of $10{ }^{\circ} \mathrm{C} \mathrm{min}^{-1}$ until a temperature of $400{ }^{\circ} \mathrm{C}$ was reached. The degree of Dex crystallinity was determined by measuring the characteristic endothermic peak of Dex at $269^{\circ} \mathrm{C}$.

\subsection{High-performance liquid chromatography}

Dex solution concentrations were determined using high-performance liquid chromatography (HPLC) (Waters 2690) and a dual absorbance UV detector (Waters 2487). The isocratic mobile phase consisted of $42 \%$ acetonitrile and $58 \%$ water with a flow rate of $1 \mathrm{ml} \mathrm{min}{ }^{-1}$. Samples of $50 \mu \mathrm{l}$ were injected directly and pumped through an Omnisphere 5 C18 Column. Dex was detected using a UV detector at $246 \mathrm{~nm}$. The retention time of Dex was $3.7 \mathrm{~min}$. Dex solution concentration was determined from a standard curve of Dex in PBS from 1 to $100 \mu \mathrm{g} \mathrm{ml}^{-1}$.

\subsection{Dex release studies}

Coatings were placed into microcentrifuge tubes containing $1.5 \mathrm{ml}$ of PBS (pH 7.4) at $37^{\circ} \mathrm{C}$. Coatings were placed into fresh $1.5 \mathrm{ml}$ PBS solutions every $24 \mathrm{~h}$ for a period of 2 weeks. Daily drug release samples were stored at $4{ }^{\circ} \mathrm{C}$ in the dark until analysis. Samples were thawed at room temperature and injected into the HPLC system and the Dex concentration was determined as described above.

To evaluate possible topographical changes due to the depletion of Dex, coatings before and after the 2 week release study were imaged by environmental scanning electron microscopy (ESEM) (wet mode). Captured images were processed as described above to calculate average thickness and pore size.

\subsection{Dex loading efficiency}

Pre- and post- gas-foamed coatings, and coatings after drug release studies were completely dissolved in $1 \mathrm{ml}$ chloroform. Solutions were then injected into the HPLC and the Dex concentration was determined as described above.

\subsection{Sensor response}

Medtronic MiniMed SOF-SENSOR ${ }^{\mathrm{TM}}$ glucose sensors, MiniLink ${ }^{\mathrm{TM}}$ transmitters, and Transmitter Utility software package were graciously supplied by Medtronic MiniMed (Northridge, CA). Bare sensors were hydrated for $2 \mathrm{~h}$ in PBS $\left(37^{\circ} \mathrm{C}\right.$, stirred) and then dipped sequentially in $100,200,400$ and $0 \mathrm{mg} \mathrm{dl}^{-1}$ glucose in PBS $\left(37^{\circ} \mathrm{C}\right.$, stirred) to obtain a calibrated baseline sensor response. Dex-loaded porous coatings were subsequently placed over the sensor tips and subjected to a glucose challenge with $100,200,400$ and $0 \mathrm{mg} \mathrm{dl}^{-1}$ of glucose. Dex-free non-porous films served as controls.

Sensor response time and signal attenuation to glucose challenge were used as metrics to evaluate sensor function. Sensor response times were calculated as the time for the sensor to achieve $90 \%$ of its steady-state calibration current for a given test interval. Attenuation was calculated as follows:

$$
\text { Attenuation } \%=\left(1-\frac{\text { Sensor Current }_{\text {Challenge Interval }}}{\text { Sensor Current }_{\text {Calibration Interval }}}\right) \times 100 \%
$$

\subsection{Peripheral blood derived human monocyte isolation}

Human monocytes were isolated from $100 \mathrm{ml}$ of EDTA-treated blood drawn from healthy volunteers $(n=12)$. Using a hypotonic density centrifugation method, buffy coats were collected from the interphase of Histopaque $1077^{\circledR}$ (Sigma, 10771). After separation, mononuclear cells were washed twice with complete RPMI 1640 medium. Subsequently, untouched monocytes were negatively selected using magnetic beads (Dynabeads ${ }^{\circledR}$, Life Technologies). Enriched monocytes were then incubated in RPMI 1640 medium supplemented with $10 \%(\mathrm{v} / \mathrm{v})$ heat-inactivated $A B$ serum, $50 \mu \mathrm{m}$ 2-mercaptoethanol, $1 \mathrm{mM}$ sodium pyruvate, $10 \mathrm{mM}$ Hepes, $4.5 \mathrm{~g} \mathrm{l}^{-1}$ glucose, and $1 \%$ antibiotic/antimycotic solution. All cultured reagents used had endotoxin levels of $<0.01 \mathrm{ng} \mathrm{ml}^{-1}$ LPS. The isolated monocyte suspension was adjusted to a concentration of $1 \times 10^{6}$ cells $\mathrm{ml}^{-1}$. The viability of the monocytes was $>95 \%$ as determined by Calcein AM intracellular stain and purity was $>90 \%$ as assessed by CD14+ marker expression using flow cytometric analysis.

\subsection{Dex-treated media preparations for apoptosis assays}

Two apoptosis studies were conducted (i) to investigate if Dex released from porous coatings retained its bioactivity after gasfoaming/salt-leaching; and (ii) to show the dose-dependent effect of Dex released from porous coatings on isolated monocytes.

For Dex bioactivity studies, $90 \%$ porous coatings loaded with $1.4 \mathrm{wt} . \%$ Dex were placed in RPMI for $24 \mathrm{~h}$ at $37^{\circ} \mathrm{C}$ allowing Dex to be released into the media, the coatings were then moved to a second set of fresh media for another $24 \mathrm{~h}$, and then to a third set of fresh media for another $24 \mathrm{~h}$. This yielded media samples with Dex released from coatings after 24, 48 and $72 \mathrm{~h}$. Dex concentrations from these solutions were determined by HPLC. Equivalent "Dex-spiked media" solutions were made by spiking RPMI media with concentrations equal to that of the Dex released from $90 \%$ 
porous coatings loaded with $1.4 \mathrm{wt} . \%$ Dex at 24,48 and $72 \mathrm{~h}$. Untreated media and media treated with Dex-free porous coatings served as controls.

For Dex dose-dependent studies, $90 \%$ porous coatings loaded with $0.7,1.4$ and 2.9 wt.\% Dex were incubated in RPMI media as described above for 24,48 and $72 \mathrm{~h}$. These solutions were then used to culture isolated monocytes. Untreated media and Dex-free porous coatings served as controls.

\subsection{Annexin-V apoptosis assays}

For both Dex apoptosis studies, monocytes were resuspended to a density of $1 \times 10^{6}$ cells ml $^{-1}$ in treated and untreated media immediately after isolation. Cells were plated in 6-well tissue culture plates. Cell samples from each well were collected every $24 \mathrm{~h}$ for a period of 3 days and cultured at $37{ }^{\circ} \mathrm{C}$ under $5 \% \mathrm{CO}_{2}$. During sample collection, cell medium was replaced by suspending monocytes to a concentration of $1 \times 10^{6}$ cells ml ${ }^{-1}$ concentration in new treated or untreated media, respectively.

Up-regulation of phosphatidylserine (PS) receptor expression was detected via Annexin- $V$ antibody staining in treated peripheral blood derived human monocytes. For staining, samples were kept in ice washed twice in staining medium (ice-cold PBS supplemented with $10 \%$ FBS and $1 \%$ sodium azide). Monocytes were directly labeled with APC-mouse $\mathrm{mAb}$ to CD14+(Invitrogen, MHCD1405) and Annexin-V FITC to PS (Invitrogen, A13199). APC and FITC-conjugated murine IgG mAbs of unrelated specificities were used as negative stain controls. Annexin- $V$ positive controls were created by treating U937 cells with camptothecin at a concentration of $4 \mu \mathrm{g} \mathrm{ml}^{-1}$ for $4 \mathrm{~h}$. After staining, suspended cells were kept in ice and protected from light until Annexin-V and CD14+ expression was analyzed by flow cytometry analysis.

\subsection{Animal studies}

\subsubsection{Preparation of implants}

Medical-grade Tygon ${ }^{\circledR}$ tubing (formulation S-54-HL Saint-Gobain, Courbevoie, France) was selected for testing of the foreign body response (FBR). Tygon ${ }^{\circledR}$ implants were of similar dimensions $(1.5 \mathrm{~cm}$ long and $0.8 \mathrm{~cm}$ outer diameter) and mechanical properties to the tips of the Medtronic MiniMed SOF-SENSOR ${ }^{\mathrm{TM}}$. Dex-free and Dex-loaded $90 \%$ porous coatings were slid onto Tygon ${ }^{\circledR}$ implants. The coatings fitted snugly over the Tygon ${ }^{\circledR}$ tubing and did not need further affixing. Bare and coated implants were sterilized with ethylene oxide and allowed to out-gas for at least 7 days prior to implantation. Bare Tygon ${ }^{\circledR}$ tubing was used as a positive control because Tygon ${ }^{\circledR}$ is a silicone known to undergo fibrous encapsulation when subcutaneously implanted [28].

\subsubsection{Implantation procedure}

All National Institutes of Health guidelines for the care and use of laboratory animals (NIH Publication No. 85-23 Rev. 1985) were observed. Approval for these studies was granted by the Institutional University Animal Care and Use Committee at Duke University prior to initiation of these studies. Sprague-Dawley rats (200-300 g; CD-type, Charles River Labs, Raleigh, NC) were shaved and prepped prior to surgery. After anesthesia induction with $3 \%$ isoflurane in oxygen, the dorsal areas were shaved and the skin prepped with chlorhexidine and alcohol three times. The rats were placed in the prone position and a sterile field was created over the dorsum. All implants were inserted via a trocar introducer. A sterile 12-gauge needle was used to access the subcutaneous plane; implants were inserted into the needle and advanced into the rat subcutis using a plunger. The needle and plunger were simultaneously drawn back while the implant stayed in the desired site. The minimal cutaneous wound did not require primary closure.

\subsubsection{Dex systemic effects}

A pilot study was conducted in order to assess the systemic in vivo effects of coated implants loaded with Dex. Porous coatings that were Dex-free or contained 0.7, 1.4 and 2.9 wt.\% Dex were implanted in the dorsal subcutis of rats ( $n=2$ for each treatment). Uncoated Tygon ${ }^{\circledR}$ tubing was also implanted. All samples were explanted 14 days post-implantation. Systemic Dex effects were monitored by noting the rat's weight and overall health every 3 days for the duration of the study, and by evaluating the tissue response to uncoated Tygon ${ }^{\circledR}$.

\subsubsection{Pilot evaluation of Dex-releasing coatings}

Samples of $90 \%$ porous coatings with $0.7 \mathrm{wt} . \%$ Dex, which did not exhibit system effects, were implanted in the dorsal subcutis of rats along with Dex-free $90 \%$ porous coatings and bare Tygon ${ }^{\circledR}$ controls. Samples were explanted 3 days post-implantation.

\subsection{Evaluation of histological samples}

Tissue samples were flash frozen using liquid nitrogen immediately upon explantation and kept at $-80^{\circ} \mathrm{C}$. Serial cryosections of $10 \mu \mathrm{m}$ thickness were cut and stained with hematoxylin and eosin (H\&E) to monitor inflammation. Cell nuclei stained purple while the connective tissue surrounding the implant stained pink.

\subsection{Statistical Analysis}

All data are presented as means \pm standard errors of the mean $( \pm S E M)$. Coating pore size, thickness and porosity results were compared using a one-way ANOVA followed by Tukey's HSD tests for all samples $(P<0.05)$. Sensor functionality and drug release were analyzed using two-way repeated-measures ANOVA followed by Tukey's HSD post hoc test $(P<0.05)$. Annexin-V apoptosis assay results were analyzed using a two-way repeated measures ANOVA. Lilliefors test on apoptosis data confirmed data normality, while a Maulchy correction was performed since the data violated Maulchy's sphericity test (unequal variances). Tukey's HSD was used for post hoc tests and the threshold for significance was $P<0.05$.

\section{Results}

\subsection{Coating pore size, thickness and porosity}

Fig. 1A shows a bare glucose sensor (left) and a sensor with a $90 \%$ porous coating (right). Coatings fit snugly over the sensor tip and were porous throughout. Fig. $1 \mathrm{~B}$ and $\mathrm{C}$ are SEM images showing the porous microstructure and coating cross-section. Fig. 2 shows micro-CT volume renderings of the solid (Fig. 2A-D) and corresponding void (Fig. 2E-H) spaces of a Dex-free $90 \%$ porous coating, and Dex-loaded $90 \%, 60 \%$ and $30 \%$ porosity coatings. Total void volume and interconnected void regions represented by individual colors in Fig. 2E-H clearly diminished with decreasing porosity.

Table 2 lists the average coating thickness and pore size measured by SEM and the coating percentage porosity calculated by micro-CT for specimens that were intended to generate porosities of $90 \%, 60 \%$ and $30 \%$. A Dex-free $90 \%$ porosity specimen was included for comparison. Coating thickness increased significantly with decreasing porogen content, but no statistical difference in average pore diameter was found across these specimen types. The average percentage porosity measured by micro-CT closely mirrored the intended specimen porosities of $90 \%, 60 \%$ and $30 \%$. The inclusion of Dex at the highest 2.9 wt.\% loading did not significantly affect pore structure, porosity or coating thickness when compared to porous Dex-free coatings. 
(A)

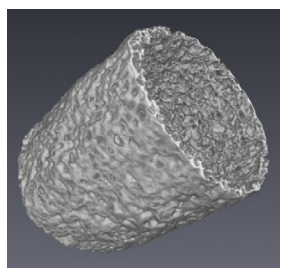

(E)

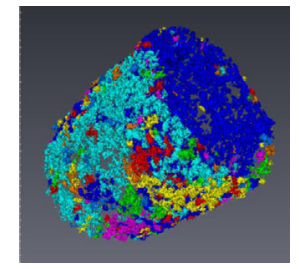

$90 \%$ Porosity - 0 wt $\%$ Dex
(B)

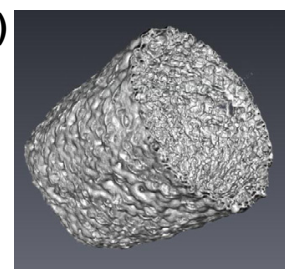

(F)

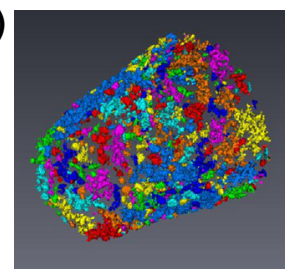

$90 \%$ Porosity -2.9 wt\% Dex
(C)

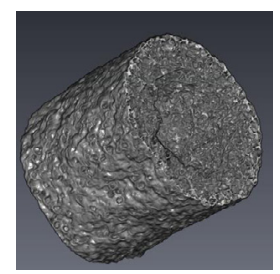

(G)

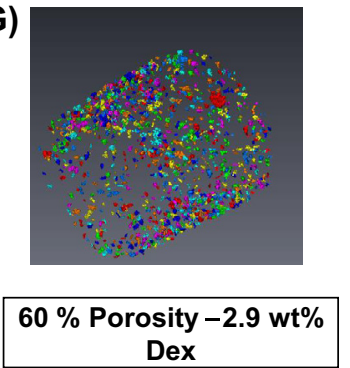

(D)

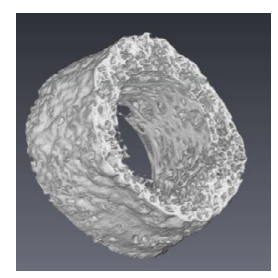

(H)

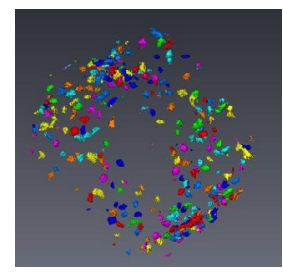

$30 \%$ Porosity -2.9 wt $\%$ Dex

Fig. 2. Representative micro-CT images of porous coatings created via the salt-leaching/gas-foaming technique with decreasing porogen fraction. The images show coatings of different morphologies created by varying the ammonium bicarbonate porogen concentration: (A,B) $90 \%$, (C) $60 \%$ and (D) $30 \%$. Addition of Dex did not disrupt scaffold structure of (A) 0 wt.\% Dex and (B-D) $2.9 \mathrm{wt}$.\%. Corresponding 3-D volume renderings of porous structures are also shown, individual colors represent interconnected void regions of coatings $(\mathrm{E}-\mathrm{H}) .(n=6$.$) .$

Table 2

Physical characteristics of porous Dex-releasing coatings $(n=6)$.

\begin{tabular}{|c|c|c|c|c|}
\hline \multicolumn{2}{|c|}{$\begin{array}{l}\text { Intended } \\
\text { composition }\end{array}$} & \multirow[t]{2}{*}{$\begin{array}{l}\text { Thickness } \\
(\mu \mathrm{m})\end{array}$} & \multirow[t]{2}{*}{$\begin{array}{l}\text { Pore diameter } \\
(\mu \mathrm{m})\end{array}$} & \multirow[t]{2}{*}{$\begin{array}{l}\text { Measured porosity } \\
(\%)\end{array}$} \\
\hline $\begin{array}{l}\text { Porosity } \\
\%\end{array}$ & $\begin{array}{l}\text { Dex } \\
\text { (wt.\%) }\end{array}$ & & & \\
\hline 90 & 0 & $85.3 \pm 7.5$ & $75.8 \pm 10.1$ & $86.2 \pm 2.6$ \\
\hline 90 & 2.9 & $80.6 \pm 8.3$ & $70.4 \pm 13.5$ & $85.4 \pm 4.1$ \\
\hline 60 & 2.9 & $130.5 \pm 9.3^{*}$ & $76.8 \pm 12.3$ & $55.7 \pm 9.8^{*}$ \\
\hline 30 & 2.9 & $205.9 \pm 17.9$ *\# & $72.8 \pm 14.9$ & $38.4 \pm 3.7^{*}$ \\
\hline
\end{tabular}

* Denotes $P<0.05$ or less compared to $90 \%$ porosity $/ 2.9$ wt.\% Dex coatings.

\# Denotes $P<0.05$ or less compared to $60 \%$ porosity/2.9 wt.\% Dex coatings.

\subsection{Differential scanning calorimetry}

DSC was employed to determine whether Dex loaded into coatings was present in a molecularly dissolved state or was sequestered as crystals. The dashed lines in Fig. 3A and B show the characteristic exothermic melting of bulk Dex crystals (dashed lines) at $269^{\circ} \mathrm{C}$. This peak was absent in all Dex-loaded polymer coatings (solid and dotted lines). These results suggest that Dex was highly soluble in the polyurethane matrix and was not sequestered in a crystallized form.

\subsection{Dex loading and retention after gas-foaming}

Table 3 lists the Dex loading before (intended) and after the salt-leaching/gas-foaming step (retained) for the coating formulations used in the Dex release studies. The wt.\% Dex retained after gas-foaming correlated directly to the initial Dex loading, while the percentage of Dex retained after the gas-foaming/salt-leaching step for all groups averaged $79.7 \pm 2.5 \%$ (mean \pm SEM).

\subsection{Dex release}

Fig. 4 presents the 2 week in vitro release profile of Dex from $90 \%$ porous coatings prepared with $0.7,1.4$ and 2.9 wt.\% Dex

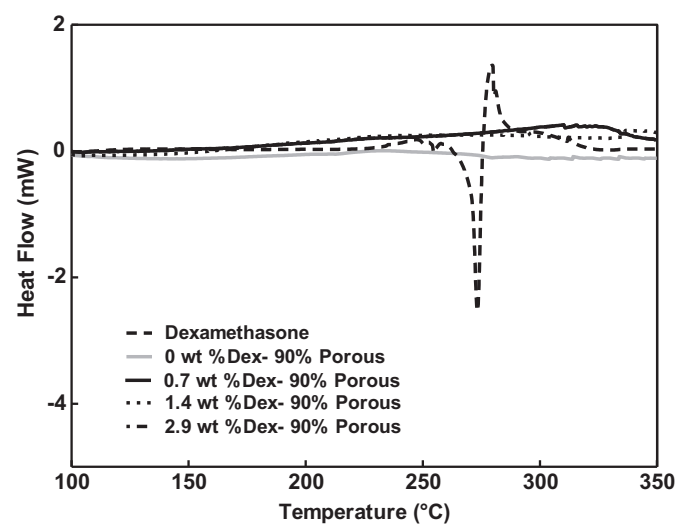

(A)

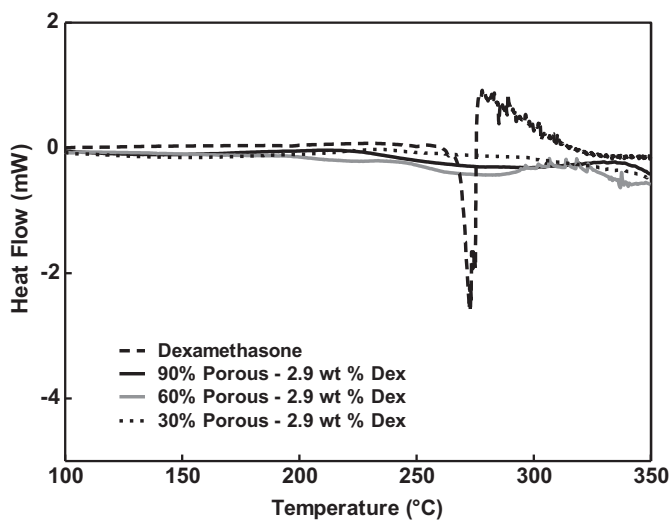

(B)

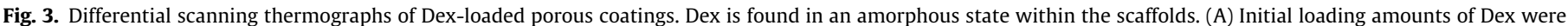

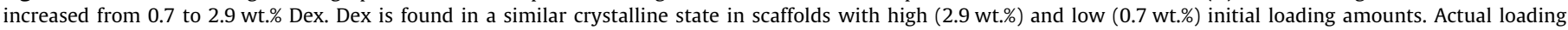
amounts are listed in Table 3. (B) Changes in coating porosity did not affect crystal state of Dex within scaffolds. ( $n=6$.). 
Table 3

Loading efficiency and release summary of Dex incorporated into porous polyurethane coatings by salt-leaching/gas-foaming method $(n=10)$.

\begin{tabular}{|c|c|c|c|c|c|c|c|}
\hline \multicolumn{2}{|c|}{$\begin{array}{l}\text { Intended sample } \\
\text { composition }\end{array}$} & \multicolumn{2}{|c|}{ Dex retained after foaming } & \multicolumn{2}{|l|}{ Average release rate ( $\mu \mathrm{g} /$ day) } & \multicolumn{2}{|c|}{$\begin{array}{l}\text { Dex retained after } 14 \text { day release } \\
\text { (wt.\%) }\end{array}$} \\
\hline Porosity \% & Dex (wt.\%) & wt.\% & $\mu \mathrm{g}$ & First week release (1-7 days) & Second week release (8-15 days) & wt.\% & $\mu \mathrm{g}$ \\
\hline 90 & 0.7 & $0.56 \pm 0.07$ & $305 \pm 35$ & $27.4 \pm 2.9$ & $7.2 \pm 1.4$ & $0.09 \pm 0.07$ & $48 \pm 38$ \\
\hline 90 & 1.4 & $1.07 \pm 0.09^{*}$ & $555 \pm 46^{*}$ & $55.7 \pm 5.9^{*}$ & $11.2 \pm 2.3$ & $0.17 \pm 0.08^{*}$ & $87 \pm 41^{*}$ \\
\hline 90 & 2.9 & $2.10 \pm 0.38^{* \#}$ & $914 \pm 163^{* \#}$ & $93.9 \pm 13.0^{* \#}$ & $15.4 \pm 6.1$ & $0.32 \pm 0.08^{* \#}$ & $138 \pm 34^{* \#}$ \\
\hline 60 & 2.9 & $2.41 \pm 0.30$ & $1195 \pm 149$ & $107.8 \pm 16.4$ & $27.2 \pm 2.9$ & $0.38 \pm 0.16$ & $189 \pm 80$ \\
\hline 30 & 2.9 & $2.51 \pm 0.32$ & $1791 \pm 230$ & $142.5 \pm 30.5$ & $32.5 \pm 3.6$ & $0.44 \pm 0.20$ & $316 \pm 143$ \\
\hline
\end{tabular}

* Denotes $P<0.05$ or less compared to 0.7 wt.\% Dex $/ 90 \%$ porosity coatings.

\# Denotes $P<0.05$ or less compared to 1.4 wt.\% Dex/90\% porosity coatings.

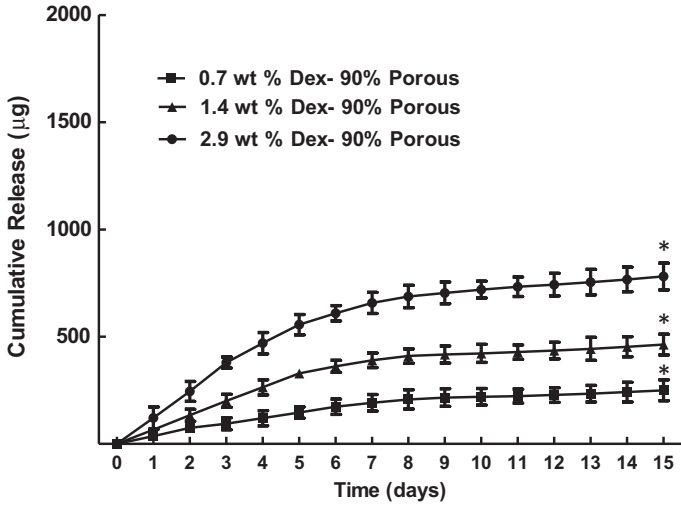

(A)

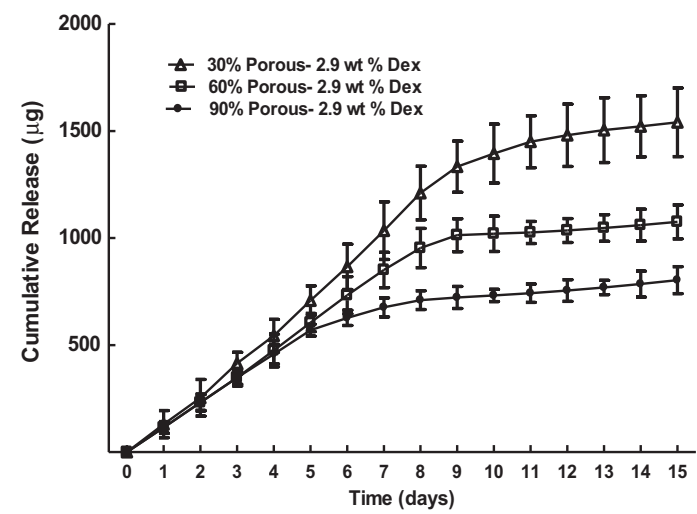

(B)

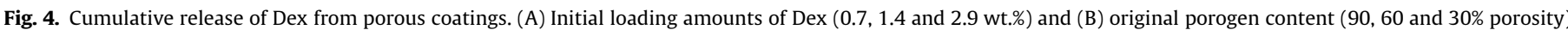
were varied. Dex release from coatings shows a high dependency on initial loading. ${ }^{*} P<0.05$ or less compared to highlighted treatments. ( $n=10$.).

loadings (Fig. 4A), and $90 \%, 60 \%$ and $30 \%$ porous coatings prepared with 2.9 wt.\% Dex loadings (Fig. 4B). Overall, the cumulative Dex release increased significantly with the amount of Dex payload. In each case, a fast release rate was observed for days 1-7, followed by a slow release rate for days 8-15 (Table 3). Release rates for days 1-7 increased with increasing Dex payload, either by increasing the wt.\% Dex for a constant percentage porosity (Fig. 4A), or by decreasing the percentage porosity for a constant wt.\% Dex (Fig. 4B). Week 2 release rates also followed the same trend but the effect was less pronounced and not significant. The last column in Table 3 lists the wt.\% Dex that remained in the coatings after 14 days of release. The percentage retained after 14 days averaged $16.0 \pm 0.9 \%$ (mean \pm SEM) over all specimens, again bearing no relationship to the percentage porosity. Moreover, SEM imaging of $90 \%$ porous coatings before and after 14 days of Dex release showed that the coating thickness and porosity was unaltered (data not shown), which one would expect for solubilized small molecule release from a non-degradable polymer.

\subsection{Sensor response time and signal attenuation}

Fig. 5A and $B$ show the in vitro responses of bare sensors and sensors fitted with porous coatings to step increases glucose concentration. Table 4 lists the corresponding sensor response times and percentage signal attenuations. All signals for sensors deployed with $90 \%$ coatings were superimposed directly over the bare sensor traces, and exhibited only slight increases in response times and signal attenuation regardless of wt.\% Dex loading. However, sensors fitted with non-porous coatings, $60 \%$ and $30 \%$ porosity films showed significantly increased response times and signal attenuation compared to base sensors. Clearly, the $90 \%$ porous coatings are best suited for use in sensors.

\subsection{Bioactivity of Dex-releasing porous coatings}

Fig. 6 displays the percentage of Annexin- $V$ positive (i.e. apoptotic) monocytes after exposure to Dex-releasing $90 \%$ porous coatings for 24, 48 and $72 \mathrm{~h}$. The $2.9 \mathrm{wt}$.\% Dex coatings significantly showed the highest percentage of Annexin- $\mathrm{V}$ positive monocytes at 24,48 and 72 h, with $25.5 \%, 49.34 \%$ and $65.5 \%$ cells being apoptotic, respectively. A significant dose dependence of wt.\% Dex at 48 and $72 \mathrm{~h}$ was observed. Apoptosis of monocytes cultured in media treated with Dex-free porous coatings was similar to the baseline level of apoptosis in untreated media. Finally, identical assays performed with Dex-spiked media exhibited equivalent levels of apoptosis as those using media from Dex released for coatings, showing that the gas-foaming step had no effect on Dex bioactivity (data not shown).

\subsection{Pilot animal testing of Dex-releasing coatings}

After 14 days, rats implanted with $2.9 \mathrm{wt} . \%$ Dex porous coatings exhibited an 11-16 wt.\% decrease as well as atrophy and thinning of the dermal tissue, whereas rats implanted with $1.4 \mathrm{wt} . \%$ Dex porous coatings did not exhibit any external signs of adverse Dex effects. However, histological examination of bare positive control implants showed an impaired inflammatory response when compared to controls from rats implanted with Dex-free coatings (data not shown). Rats implanted with $0.7 \mathrm{wt} . \%$ Dex porous coatings were able to mount an appropriate immune response to positive control implants, and showed no external signs of systemic Dex 


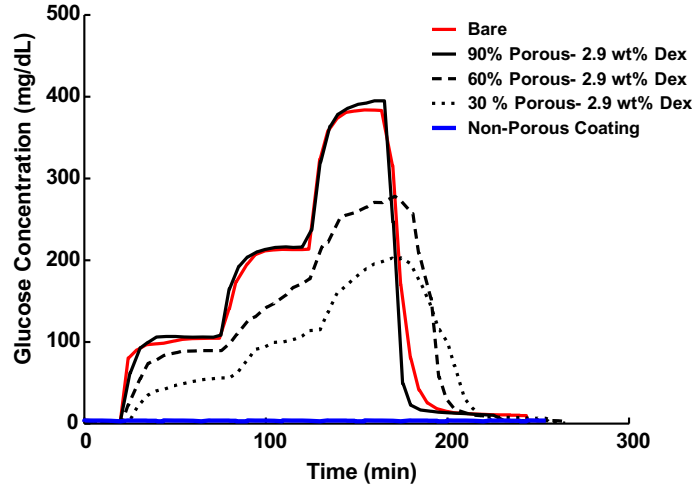

(A)

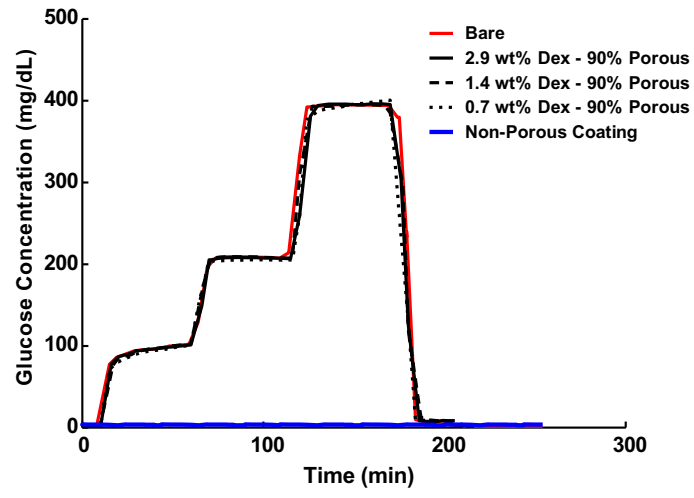

(B)



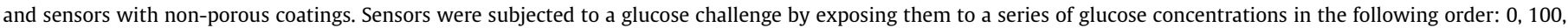

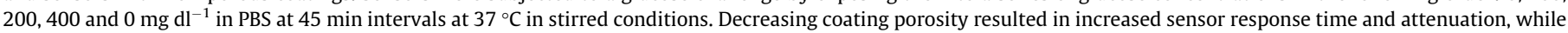
changes in Dex loading did not affect the sensor signal when compared to bare sensors. Data presented are representative traces of six independent tests $(n=6)$.

Table 4

In vitro response of sensors deployed with Dex-releasing polyurethane porous coatings $(n=6)$

\begin{tabular}{|c|c|c|c|c|c|c|}
\hline \multicolumn{2}{|c|}{ Sample composition } & \multicolumn{4}{|c|}{ Time (min) to reach $90 \%$ steady state current } & \multirow[t]{2}{*}{$\%$ Attenuation $400 \rightarrow 0^{\mathrm{a}}$ Interva } \\
\hline Porosity \% & Dex (wt.\%) & $0 \rightarrow 100^{\mathrm{a}}$ & $100 \rightarrow 200^{\mathrm{a}}$ & $200 \rightarrow 400^{\mathrm{a}}$ & $400 \rightarrow 0^{\mathrm{a}}$ & \\
\hline Bare sensor & & $5 \pm 2$ & $7 \pm 2$ & $10 \pm 4$ & $15 \pm 6$ & $6.5 \pm 1.8$ \\
\hline 90 & 2.9 & $8 \pm 3$ & $7 \pm 3$ & $17 \pm 3$ & $24 \pm 7$ & $8.6 \pm 1.9$ \\
\hline 60 & 2.9 & $17 \pm 10$ & $>45^{\mathrm{b}^{* \#}}$ & $>45^{\mathrm{b}^{* \#}}$ & $>45^{\mathrm{b} * \#}$ & $24.8 \pm 6.9$ \\
\hline 30 & 2.9 & $>45^{\mathrm{b} * \# \&}$ & $>45^{\mathrm{b}^{* \#}}$ & $>45^{\mathrm{b}^{* \#}}$ & $>45^{\mathrm{b}^{*} \#}$ & $49.7 \pm 13.4^{* \#}$ \\
\hline \multicolumn{2}{|c|}{ Non-porous coating } & $>45^{\mathrm{b} * \# \&}$ & $>45^{\mathrm{b} * \#}$ & $>45^{\mathrm{b}^{* \#}}$ & $>45^{\mathrm{b}^{*} \#}$ & $92.5 \pm 3.5^{* \#}$ \\
\hline
\end{tabular}

${ }^{\text {a }}$ Glucose challenge ( $\mathrm{mg} \mathrm{dl}^{-1}$ ).

b Time exceeded experimental interval.

* Denotes $P<0.05$ or less compared to bare sensor.

\# Denotes $P<0.05$ or less compared to $90 \%$ Porosity/2.9 wt\% Dex coatings.

\& Denotes $P<0.05$ or less compared to $60 \%$ porosity $/ 2.9$ wt.\% Dex coatings.

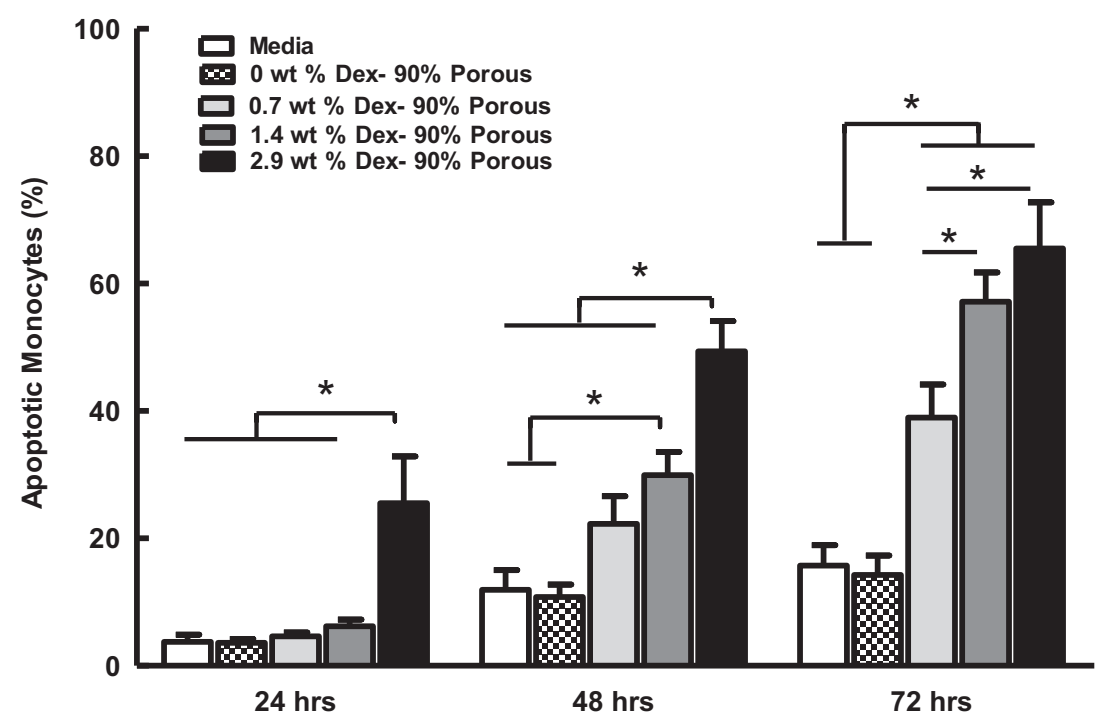

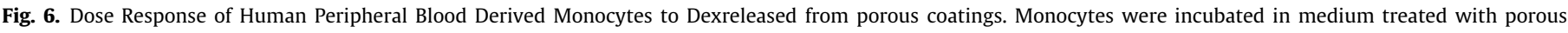




$(n=8)$.

effects. From this study it was determined that $0.7 \mathrm{wt} . \%$ Dex porous coatings were the most fitting for further in vivo tests in rats.

Fig. 7 shows low- and high-magnification images of day $3 \mathrm{H} \& \mathrm{E}-$ stained sections of tissue that surrounded Tygon tubing that was either bare or had $90 \%$ porous coatings with either 0.7 or 0 wt.\% Dex. The Dex-loaded coatings showed decreased immune cell infiltration to the implant site when compared to Dex-free porous coatings and the bare controls. Moreover, the acute inflammatory 



(A)
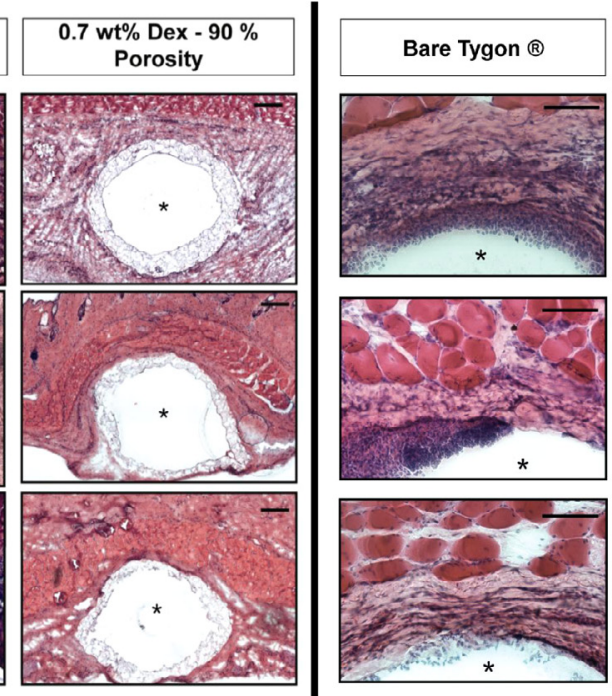
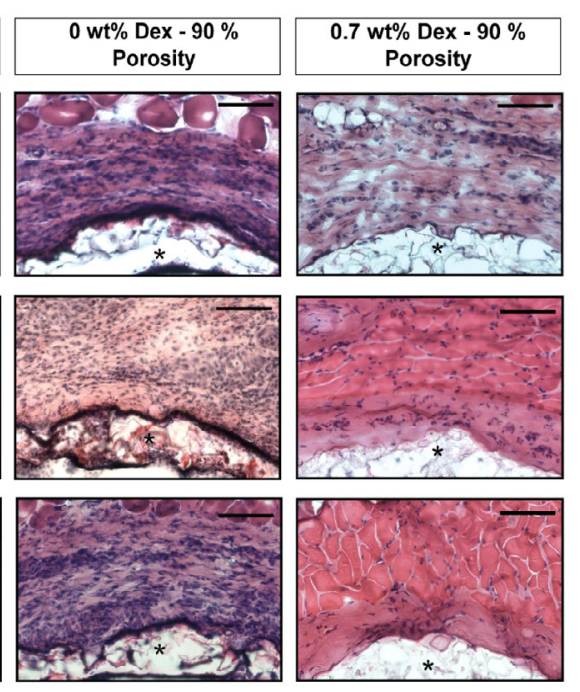

(B)

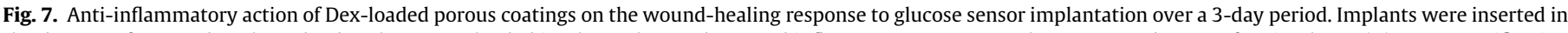

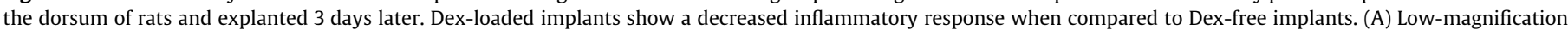

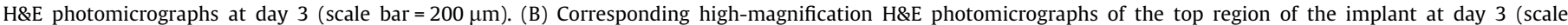
$\operatorname{bar}=100 \mu \mathrm{m})$. $(n=3$. $)$

response to Dex-free porous coatings was comparable in magnitude to that of the positive Tygon ${ }^{\circledR}$ controls.

\section{Discussion}

Tissue-associated anomalies with implanted glucose sensors have been an area of concern for many years. These effects can begin immediately following implantation or can arise during the "break-in period" that occurs during the first few days following sensor insertion. During this time, the sensor is presented with an unstable microenvironment characterized by immune cell infiltration, inflammation and formation of a granulation tissue. Strategies for improving in vivo sensor performance include development of new biomaterials and localized drug delivery for resisting biofouling, attenuating inflammation, and increasing vascularization of the foreign body capsule [17,25,26,29].

Previously, we reported porous polyester coatings deployed at the tips of needle-type glucose sensors that increased vascularization and perfusion of the tissue-sensor interface; however, implanted sensors succumbed to inflammation and immune cell infiltration, resulting in early and pronounced signal reduction [27]. We now employ a segmented polyurethane in order to release the moderately hydrophobic anti-inflammatory glucocorticoid Dex from the porous coatings. The present study characterized the physical properties and drug-releasing properties of the porous coatings, the bioactivity of the Dex released from porous coatings, and the effect of these porous coatings on sensor performance.

The porous Dex-loaded Tecoflex ${ }^{\circledR}$ 93A coatings described herein have a tunable microstructure. Pore size was controlled by sieving ammonium bicarbonate particulates to a desired size range of 50$75 \mu \mathrm{m}$. Immersing particulate-containing polymer coatings in a heated water bath generated pores with sizes in the upper end of the sieved salt particle size range (Table 2). Micro-CT generated void volume renderings showed a mostly interconnected pore structure that was most pronounced in coatings with high porogen content (Fig 2). Moore et al. similarly demonstrated that increasing the concentration of leachable sodium chloride particulates in polyester scaffolds for bone tissue engineering resulted in increased scaffold void volume and pore interconnectivity [30,31].

The poration step caused between $13 \%$ and $28 \%$ of the originally loaded Dex to be lost from the polymer coating (Table 4). The largest Dex loss occurred for the specimens with the highest polymer surface area and wt.\% Dex payload (90\% porosity and 2.9 wt.\% Dex). Dex loss during poration can be attributed to increased specimen porosity, the steep concentration gradient imposed on the coatings during salt-leaching/gas-foaming fabrication steps, and heatinduced enhanced drug and polymer chain mobility [32]. DSC showed that there was no evidence of crystallized Dex present in porous coatings (Fig. 3), indicating that Dex was molecularly dissolved within the polyurethane matrix.

Dex-loaded porous coatings exhibited a fast initial release followed by a slower secondary release characteristic of monolithic drug delivery from a polymer matrix (Fig 4). Daily Dex release from coatings over the 15-day release period was within the therapeutic rage for the treatment of localized acute inflammation (Table 3 ). Interestingly, changes in coating porosity (surface area) did not significantly affect drug release rates. Therefore, the high drug payload of Dex over a short delivery window (2 weeks) cannot be attributed to just simple diffusion of a small molecule drug through a thin and highly porous surface $[33,34]$. Tecoflex ${ }^{\mathbb{R}} 93 \mathrm{~A}$ is a segmented polyurethane comprised of soft microdomains of poly(tetramethylene oxide) and hard crystalline microdomains of bis(4-isocyanatocyclohexyl)methane $\left(\mathrm{H}_{12} \mathrm{MDI}\right)$ and 1,4-butanediol. As a non-polar drug, Dex preferentially interacts with the amorphous soft microdomains of the polyurethane; these regions serve as drug reservoirs while empty hard crystalline microdomains act as channels for drug release [35,36]. Similar results were reported by Gupta et al. [33], where the small molecule hydrophobic drug, dapivirine, was released from Tecoflex ${ }^{\circledR}$ rings for vaginal drug delivery applications. This study also showed that Dex release from the rings was fast and did not follow first-order release kinetics.

In vitro sensor responses to glucose challenge studies (Table 4 and Fig. 5) showed that sensor response was not hindered by $90 \%$ porous coatings. Signal lag and attenuation in low-porosity coatings can be attributed to the increased thickness, decreased pore interconnectivity and reduced total void volume. Functional 
sensor studies by Koshwanez et al. [27] similarly showed that sensors with highly porous thick coatings $(>200 \mu \mathrm{m})$ had elevated response times and diminished signal when compared to bare sensors. This effect was ascribed to the augmented migration distance required for glucose to reach the sensor surface. Dex release from coatings also did not interfere with sensor functionality (Fig. 5b). Studies by other groups utilizing glucose oxidase based sensors did not show signal fluctuations while functioning within the presence of locally released Dex $[11,37,38]$. Based on signal attenuation and lag times, only $90 \%$ porous coatings were used for in vitro Dex bioactivity and dose response.

The anti-inflammatory mechanism of Dex has been mostly associated with regulation of cytokine production and reduced metabolic activity of immune cells [24]; however, it has recently been highlighted that Dex may also control inflammatory and repair tissue responses by preferentially inducing apoptosis of active inflammatory cells (monocytes, macrophages, T cells), while protecting against apoptotic signals in cells involved in tissue repair (epithelial cells and fibroblast) $[21,39]$. Consequently the apoptotic monocyte assay reported by Schmidt et al. [21] was used to show that Dex released from $90 \%$ porous coatings had a dosedependent therapeutic effect on immune cells in culture, and that this effect was retained after the heat processing during the gasfoaming/salt-leaching step.

In the Schmidt assay the Annexin-V protein preferentially binds to the PS receptor, which is externalized on the surface during early cell apoptosis. Annexin-V binding can therefore be used as a marker for apoptosis detection on individual cells. Dex released from coatings proved to be capable of inducing apoptosis in a time- and dose-dependent manner (Fig. 6). Both increasing the Dex payload of coatings and increasing the exposure time to Dex increased the number of monocytes positive for Annexin-V. Media samples made from Dex-free porous coatings also did not show enhanced Annexin- $V$ uptake, highlighting the role of Dex as an apoptotic regulator of peripheral blood derived human monocytes.

Pilot animal studies were performed to determine whether porous coatings with payloads of $0.7,1.4$ and 2.9 wt.\% Dex would elicit an anti-inflammatory repose in rodents without any apparent system effects. Not surprisingly, highly porous coatings with $0.7 \mathrm{wt} . \%$ Dex loading were best suited for in vivo deployment since they were able to suppress inflammation locally, while still allowing for adequate immune function at distant sites. This formulation initially delivers $\sim 30 \mu \mathrm{g}$ per day of Dex (Table 3 ), which is within the range of the recommended effective Dex daily dosage [11].

Coatings were further tested in a 3-day pilot study to determine Dex's effects on wound healing. Tygon ${ }^{\circledR}$ tubing was chosen for implantation instead of non-functional glucose sensors because hydrogen peroxide builds up in the subcutaneous space adjacent to the glucose oxidase present on non-functional sensors. In fully functional sensors, the applied voltage quickly breaks down the hydrogen peroxide generated by glucose oxidase before it can accumulate. In our study, implant sites with Dex-free porous coatings and bare Tygon ${ }^{\circledR}$ controls showed a strong inflammatory responses with dense fields of polymorphonuclear leukocytes, monocytes and macrophages surrounding the implants. In contrast, Dex-loaded coatings showed a noticeably attenuated response with fewer immune cells surrounding the implant. These results demonstrate that Dex released from coatings is not only bioactive but also capable of mediating the immune response to sensor implantation.

Prior studies have shown that local Dex delivery is able to improve sensor performance past the "break-in period" by decreasing leukocyte migration and inflammation at the implant site $[19,22,23,25,38]$. However, when Dex is delivered over the long-term ( $>1$ month) the drug can act as an angiostatic agent leading to reduced microvessel density, vasodilation, and increased vascular permeability at the implant site.

The textured coatings presented delivered the majority of the Dex payload over the initial week in vitro. This release is suitable to take sensors though the break-in period, while the topographical cues presented by the textured surface are intended to activate endothelial cells to start the neovascularization process. Although we demonstrated the short-term in vivo anti-inflammatory effect of coatings, more exhaustive in vivo testing is necessary to fully understand their potential as a platform to extend and improve the functionality of implanted sensors.

The findings reported in this study show a system capable of modulating the early stages of foreign body response to an implant. Nevertheless, the integration of Dex-releasing porous coatings into commercially available glucose-sensing platforms faces significant regulatory challenges. As a combination product, the system would require rigorous testing before it can reach the diabetic patient population [40]. Commercial enterprises have in turn resorted to the use of novel algorithms and recalibration schemes to extend the in vivo functionality of continuous glucose sensors $[41,42]$.

Glucose-sensing systems have on occasion reported measurements for up to several months in vivo [43]; however, glucose readings from these studies are still heavily dependent on numerical analysis and not sufficiently accurate clinically to pass regulatory approval and cannot be used as the sole means for programed insulin delivery $[44,45]$. Therefore, the implementation of biomaterial and drug-delivery strategies to extend sensor function should continue to be explored. Approaches such as localized Dex release and delivery of topographical cues from the sensor surface could drive the generation of a desirable tissue microenvironment that could result in extended in vivo sensor functionality.

\section{Conclusions}

Highly porous Dex-loaded coatings were fabricated by the gasfoaming/salt-leaching technique. The coatings had a controlled pore size and interconnected microstructure. A therapeutic dose of Dex was loaded into coatings and proved to be in a molecularly dissolved state. Dex release from coatings showed a typical initial fast release followed by a steady release with a high dependency on drug loading over a 15-day period. Porosity did not affect overall Dex release kinetics; however, decreasing coating porosity increased sensor signal lag-time and attenuation. Therefore, $90 \%$ porous Dex-releasing coatings were determined to be best for bioactivity testing. Dex released from coatings was able to induced apoptosis of human-derived peripheral blood monocytes in a timeand dose-dependent manner. A pilot animal study over a 3-day period confirmed that Dex released from coatings is capable of mediating the acute inflammatory response of tissue surrounding implants. Future work will focus on further in vivo testing of these coatings, which will allow us to fully assess if the combinatorial strategy of anti-inflammatory drug release and delivery of topographical cues from coatings could encourage tissue ingrowth and angiogenesis while reducing the immune response to implanted sensor leads.

\section{Acknowledgments}

This work was funded by NIH grant DK 54932 (W.M.R.). We gratefully acknowledge Medtronic Inc. for kind donation of Mini-

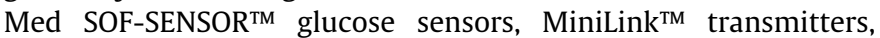
Transmitter Utility software package, and for a Medtronic Graduate Fellowship (S.V.H.). The authors would like to thank our Duke Colleagues: Charles S. Wallace for helpful technical discussions, 
Michelle Gignac, James Thostenson for assistance with the SEM and micro-CT instruments; Jinny Cho and Alina Bocio for assistance with histological stains, and members of the Needham Lab for assistance with DSC. We also thank Patrick Kaiser from the University of Utah, and Lubrizol, Inc. for donation of Tecoflex ${ }^{\circledR}$ polymers.

\section{Appendix A. Figures with essential color discrimination}

Certain figures in this article, particularly Figs. 1, 2, 5 and 7 are difficult to interpret in black and white. The full color images can be found in the on-line version, at http://dx.doi.org/10.1016/ j.actbio.2014.07.019.

\section{References}

[1] Jaremko J, Rorstad O. Advances toward the implantable artificial pancreas for treatment of diabetes. Diabetes Care 1998;21(3):444-50.

[2] Klonoff DC. Continuous glucose monitoring roadmap for 21 st century diabetes therapy. Diabetes Care 2005;28(5):1231-9.

[3] Le NN, Rose MB, Levinson H, Klitzman B. Interstitial fluid physiology as it relates to glucose monitoring technologies: implant healing in experimental animal models of diabetes. J Diabetes Sci Technol 2011;5(3):605.

[4] Mastrototaro JJ. The MiniMed continuous glucose monitoring system. Diabetes Technol Ther 2000;2(1, Supplement 1):13-8.

[5] Reach G, Wilson GS. Can continuous glucose monitoring be used for the treatment of diabetes? Anal Chem 1992:64(6):381A-6A.

[6] Nichols SP, Koh A, Storm WL, Shin JH, Schoenfisch MH. Biocompatible materials for continuous glucose monitoring devices. Chem Rev 2013;113(4):2528-49.

[7] Wisniewski N, Reichert M. Methods for reducing biosensor membrane biofouling. Colloids Surf B 2000;18(3):197-219.

[8] Koschwanez HE, Reichert WM. In vitro, in vivo and post explantation testing of glucose-detecting biosensors: current methods and recommendations. Biomaterials 2007;28(25):3687-703.

[9] Anderson JM, Rodriguez A, Chang DT, editors. Foreign body reaction to biomaterials. Seminars in immunology. Amsterdam: Elsevier; 2008

[10] Quinn CA, Connor RE, Heller A. Biocompatible, glucose-permeable hydrogel for in situ coating of implantable biosensors. Biomaterials 1997;18(24):1665-70.

[11] Hickey T, Kreutzer D, Burgess D, Moussy F. In vivo evaluation of a dexamethasone/PLGA microsphere system designed to suppress the inflammatory tissue response to implantable medical devices. J Biomed Mater Res 2002;61(2):180-7.

[12] Novak MT, Yuan F, Reichert WM. Predicting glucose sensor behavior in blood using transport modeling: relative impacts of protein biofouling and cellular metabolic effects. J Diabetes Sci Technol 2013;7(6).

[13] Klueh U, Frailey JT, Qiao Y, Antar O, Kreutzer DL. Cell based metabolic barriers to glucose diffusion: macrophages and continuous glucose monitoring. Biomaterials 2014;35(10):3145-53.

[14] Klueh U, Qiao Y, Frailey JT, Kreutzer DL. Impact of macrophage deficiency and depletion on continuous glucose monitoring in vivo. Biomaterials 2014:35(6):1789-96.

[15] Fiorentino DF, Zlotnik A, Mosmann T, Howard M, O'garra A. IL-10 inhibits cytokine production by activated macrophages. J Immunol 1991:147(11):3815-22.

[16] Brown BN, Ratner BD, Goodman SB, Amar S, Badylak SF. Macrophage polarization: an opportunity for improved outcomes in biomaterials and regenerative medicine. Biomaterials 2012;33(15):3792-802.

[17] Nichols SP, Koh A, Brown NL, Rose MB, Sun B, Slomberg DL, et al. The effect of nitric oxide surface flux on the foreign body response to subcutaneous implants. Biomaterials 2012;33(27):6305-12.

[18] Corry D, Moran J. Assessment of acrylic bone cement as a local delivery vehicle for the application of non-steroidal anti-inflammatory drugs. Biomaterials 1998;19(14):1295-301.

[19] Ward WK, Hansen JC, Massoud RG, Engle JM, Takeno MM, Hauch KD. Controlled release of dexamethasone from subcutaneously-implanted biosensors in pigs: localized anti-inflammatory benefit without systemic effects. J Biomed Mater Res, Part A 2010;94A(1):280-7. http://dx.doi.org/ 10.1002/ibm.a.32651.

[20] Klueh U, Kaur M, Montrose DC, Kreutzer DL. Inflammation and glucose sensors: use of dexamethasone to extend glucose sensor function and life span in vivo. J Diabetes Sci Technol 2007:1(4):496-504.

[21] Schmidt M, Pauels H-G, Lügering N, Lügering A, Domschke W, Kucharzik T. Glucocorticoids induce apoptosis in human monocytes: potential role of IL-1 $\beta$. J Immunol 1999;163(6):3484-90.
[22] Norton L, Tegnell E, Toporek S, Reichert W. In vitro characterization of vascular endothelial growth factor and dexamethasone releasing hydrogels for implantable probe coatings. Biomaterials 2005;26(16):3285-97.

[23] Klueh U, Kaur M, Montrose DC, Kreutzer DL. Inflammation and glucose sensors: use of dexamethasone to extend glucose sensor function and life span in vivo. J Diabetes Sci Technol (Online) 2007;1(4):496.

[24] Van der Velden V. Glucocorticoids: mechanisms of action and antiinflammatory potential in asthma. Mediators Inflamm 1998;7(4):229-37.

[25] Norton L, Koschwanez H, Wisniewski N, Klitzman B, Reichert W. Vascular endothelial growth factor and dexamethasone release from nonfouling sensor coatings affect the foreign body response. J Biomed Mater Res, Part A 2007;81(4):858-69.

[26] Koschwanez H, Reichert W, Klitzman B. Intravital microscopy evaluation of angiogenesis and its effects on glucose sensor performance. J Biomed Mate Res, Part A 2010;93(4):1348-57.

[27] Koschwanez H, Yap F, Klitzman B, Reichert W. In vitro and in vivo characterization of porous poly-L-lactic acid coatings for subcutaneously implanted glucose sensors. J Biomed Mater Res, Part A 2008;87(3):792-807.

[28] James SJ, Pogribna M, Miller BJ, Bolon B, Muskhelishvili L. Characterization of cellular response to silicone implants in rats: implications for foreign-body carcinogenesis. Biomaterials 1997;18(9):667-75.

[29] Novak MT, Yuan F, Reichert WM. Modeling the relative impact of capsular tissue effects on implanted glucose sensor time lag and signal attenuation. Anal Bioanal Chem 2010;398(4):1695-705.

[30] Moore MJ, Jabbari E, Ritman EL, Lu L, Currier BL, Windebank AJ, et al Quantitative analysis of interconnectivity of porous biodegradable scaffolds with micro-computed tomography. J Biomed Mater Res, Part A 2004;71(2):258-67.

[31] Otsuki B, Takemoto M, Fujibayashi S, Neo M, Kokubo T, Nakamura T. Pore throat size and connectivity determine bone and tissue ingrowth into porous implants: three-dimensional micro-CT based structural analyses of porous bioactive titanium implants. Biomaterials 2006;27(35):5892-900.

[32] Yoon JJ, Kim JH, Park TG. Dexamethasone-releasing biodegradable polyme scaffolds fabricated by a gas-foaming/salt-leaching method. Biomaterials 2003;24(13):2323-9.

[33] Gupta KM, Pearce SM, Poursaid AE, Aliyar HA, Tresco PA, Mitchnik MA, et al. Polyurethane intravaginal ring for controlled delivery of dapivirine, a nonnucleoside reverse transcriptase inhibitor of HIV-1. J Pharm Sci 2008;97(10):4228-39.

[34] Johnson TJ, Gupta KM, Fabian J, Albright TH, Kiser PF. Segmented polyurethane intravaginal rings for the sustained combined delivery of antiretroviral agents dapivirine and tenofovir. Eur J Pharm Sci 2010;39(4):203-12.

[35] Yui N, Kataoka K, Sakurai Y, Katono H, Sanui K, Ogata N. Novel design of microreservoir-dispersed matrices for drug delivery formulations: drug release from polybutadiene- and poly (ethylene oxide)-based segmented polyurethanes in relation to their microdomain structures. J Bioact Compat Polym 1988;3(2):106-25. http://dx.doi.org/10.1177/088391158800300202.

[36] Yui N, Kataoka K, Yamada A, Sakurai Y, Sanui K, Ogata N. Drug release from monolithic devices of segmented polyether-poly (urethane-urea)s having both hydrophobic and hydrophilic soft segments, Die Makromolekulare Chemie. Rapid Commun 1986;7(11):747-50.

[37] Ju YM, Yu B, West L, Moussy Y, Moussy F. A novel porous collagen scaffold around an implantable biosensor for improving biocompatibility. II. Long-term in vitro/in vivo sensitivity characteristics of sensors with NDGA- or GAcrosslinked collagen scaffolds. J Biomed Mater Res, Part A 2010;92(2):650-8.

[38] Ju YM, Yu B, West L, Moussy Y, Moussy F. A dexamethasone-loaded PLGA microspheres/collagen scaffold composite for implantable glucose sensors. Biomed Mater Res, Part A 2010;93(1):200-10.

[39] Amsterdam A, Tajima K, Sasson R. Cell-specific regulation of apoptosis by glucocorticoids: implication to their anti-inflammatory action. Biochen Pharmacol 2002:64(5):843-50.

[40] Foote SB, Berlin RJ. Can regulation be as innovative as science and technologythe FDA's regulation of combination products. Minn J Law Sci Technol 2004;6:619.

[41] Gough DA, Kumosa LS, Routh TL, Lin JT, Lucisano JY. Function of an implanted tissue glucose sensor for more than 1 year in animals. Sci Transl Med 2010;2(42). 42ra53-42ra53.

[42] Garcia A, Rack-Gomer AL, Bhavaraju NC, Hampapuram H, Kamath A, Peyser T, et al. Dexcom G4AP: an advanced continuous glucose monitor for the artificial pancreas. J Diabetes Sci Technol 2012;7(6):1436-45.

[43] Gilligan BC, Shults M, Rhodes RK, Jacobs PG, Brauker JH, Pintar TJ, et al Feasibility of continuous long-term glucose monitoring from a subcutaneous glucose sensor in humans. Diabetes Technol Ther 2004;6(3):378-86.

[44] Clarke WL, Kovatchev B. Sensors \& algorithms for continuous glucose monitoring: continuous glucose sensors: continuing questions about clinical accuracy. J Diabetes Sci Technol (Online) 2007;1(5):669.

[45] Clarke WL, Kovatchev B. Continuous glucose sensors: continuing questions about clinical accuracy. J Diabetes Sci Technol 2007:1(5):669-75. 